\title{
Non-empirical Prediction of the Photophysical and Magnetic Properties of Systems with Open d- and f-Shells Based on Combined Ligand Field and Density Functional Theory (LFDFT)
}

\author{
Claude Daul ${ }^{\star}$
}

\begin{abstract}
Despite the important growth of ab initio and computational techniques, ligand field theory in molecular science or crystal field theory in condensed matter offers the most intuitive way to calculate multiplet energy levels arising from systems with open shells $d$ and/or $f$ electrons. Over the past decade we have developed a ligand field treatment of inorganic molecular modelling taking advantage of the dominant localization of the frontier orbitals within the metal-sphere. This feature, which is observed in any inorganic coordination compound, especially if treated by Density Functional Theory calculation, allows the determination of the electronic structure and properties with a surprising good accuracy. In ligand field theory, the theoretical concepts consider only a single atom center; and treat its interaction with the chemical environment essentially as a perturbation. Therefore success in the simple ligand field theory is no longer questionable, while the more accurate molecular orbital theory does in general over-estimate the metal-ligand covalence, thus yields wave functions that are too delocalized. Although LF theory has always been popular as a semi-empirical method when dealing with molecules of high symmetry e.g. cubic symmetry where the number of parameters needed is reasonably small ( 3 or 5), this is no more the case for molecules without symmetry and involving both an open $\mathrm{d}$ - and f-shell (\# parameters $\sim 90)$. However, the combination of LF theory and Density Functional (DF) theory that we introduced twenty years ago can easily deal with complex molecules of any symmetry with two and more open shells. The accuracy of these predictions from $1^{\text {st }}$ principles achieves quite a high accuracy $(<5 \%)$ in terms of states energies. Hence, this approach is well suited to predict the magnetic and photo-physical properties arbitrary molecules and materials prior to their synthesis, which is the ultimate goal of each computational chemist. We will illustrate the performance of LFDFT for the design of phosphors that produces light similar to our sun and predict the magnetic anisotropy energy of single ion magnets.
\end{abstract}

Keywords: Density Functional Theory · Ligand Field Theory · Phosphors · Single ion magnets · Warm-white light

\section{Introduction}

An historical consideration of ligand field theory can be obtained from ref. [1]. Briefly, this theoretical model was introduced by John Hasbrouck van Vleck more than eighty years ago, almost simultaneously to the crystal field theory of Hans Bethe. ${ }^{[1]}$ It was a semi-empirical concept operating with adjustable parameters. The ligand field theory has also been reviewed during this time. We consider especially the Angular Overlap Model (AOM) of Christian Klixbüll Jorgensen and Klaus Erik Schäffer ${ }^{[2]}$ being able to bring more chemical insight on the ligand field concept. The AOM still works with adjustable parameters but also takes into account the

\footnotetext{
${ }^{\star}$ Correspondence: Prof. C. Daul

Computational Chemistry Lab

University of Fribourg

Switzerland

E-mail: claude.daul@unifr.ch
}

angular geometry of the metal complex. Given the important growth of computational techniques, twenty years ago we have operated ligand field theory in a non-empirical way by proposing a theoretical model based on Density Functional Theory. ${ }^{[3]}$ We named this model LFDFT, as the combination of LF for ligand field and DFT for Density Functional Theory. The purpose of the model itself consists in the explicit treatment of near degeneracy correlation using ad hoc Configuration Interaction (CI) within the active space of Kohn-Sham (KS) orbitals with dominant d-and/or f-character. ${ }^{[1]}$ This CI treatment is based on a symmetry decomposition in the full rotation group and/or on a ligand field analysis of the energies of all the single determinant (micro-states) arising from this active space computed by means of DFT. The computational innovation at this stage of the development of the model consists to the formulation of the multi-determinental concept in DFT using the approach of the average of configuration (AOC) type calculation, ${ }^{[3]}$ i.e. the active space is fundamentally distinguished within an AOC occupation of frozen Kohn-Sham-orbitals (KS), eventually with fractional occupations of the d- or f-orbitals. In principle the proposed model determines the multiplet energy levels with an accuracy of a few hundred wave numbers as well as the fine structure splitting accurate to less than a tenth of this amount. Therefore the model has already given satisfactory results for some customary molecular properties such as Zero Field Splitting (ZFS), ${ }^{[4]}$ Zeeman interaction, ${ }^{[5]}$ Hyper-Fine Splitting (HFS), ${ }^{[5]}$ magnetic exchange coupling, ${ }^{[6]}$ shielding constants, ${ }^{[7]}$ etc.

Recently the LFDFT method has been extended to handle two-open-shell systems as it is important in the understanding of the optical manifestations of lanthanide phosphors. ${ }^{[8]}$ 
We proceed next to a concise description of the LFDFT methodology and finally a couple of typical applications.

\section{The LFDFT Approach}

Stricto sensus, ligand field theory is nothing but the consideration of active $\mathrm{d}$ - and/or f-electrons moving in the potential of a passive chemical environment. Wesolowski and Warshel,[9] using the concept of Frozen-Density-Embedding (FDE), gave a rigorous formulation of this:

$$
\begin{aligned}
& V_{e m b}(\mathbf{r})=\int \frac{\rho_{L}\left(\mathbf{r}^{\prime}\right)}{\left|\mathbf{r}-\mathbf{r}^{\prime}\right|} d \mathbf{r}^{\prime}+V_{X C}\left[\rho_{M}(\mathbf{r})\right. \\
& \left.+\rho_{L}(\mathbf{r})\right]-V_{X C}\left[\rho_{M}(\mathbf{r})\right]+\frac{\delta T_{s}^{\text {nadd }}\left[\rho_{M}, \rho_{L}\right]}{\delta \rho_{M}}(1)
\end{aligned}
$$

where the symbols used in Eqn. (1) are self-explanatory.

In the formulation of the FDE (Eqn. (1)) only the first term is known, for the residual terms approximations are needed. However, the first term corresponds exactly to the approach made by Bethe and Van Vleck when they developed their ligand field model.[1] In the ligand field model, the action of the chemical environment through an electrostatic perturbation can be represented in the following:

$$
\mathbf{h}=\mathbf{h}_{\mathbf{o}}+v_{L F}
$$

where $\mathbf{h}_{\mathbf{0}}$ is the Hamiltonian proper to the free ion comprising the interactions due to the kinetic energy, the nuclear attraction and the spin-orbit coupling of the electrons; and $v_{L F}$ is the electrostatic potential exerted by the ligand charge density at the metal center. If $\rho(\mathbf{r})$ is the charge density due to the ligands together with the whole chemical environment, one can formulate the electrostatic potential in the following:

$$
v_{L F}(\mathbf{r})=-e \int \frac{\rho(\mathbf{R})}{|\mathbf{R}-\mathbf{r}|} d \mathbf{R}
$$

where $e$ represents the charge of the electron and the rest of the symbols used in Eqn. (3) is graphically defined in Fig. 1.

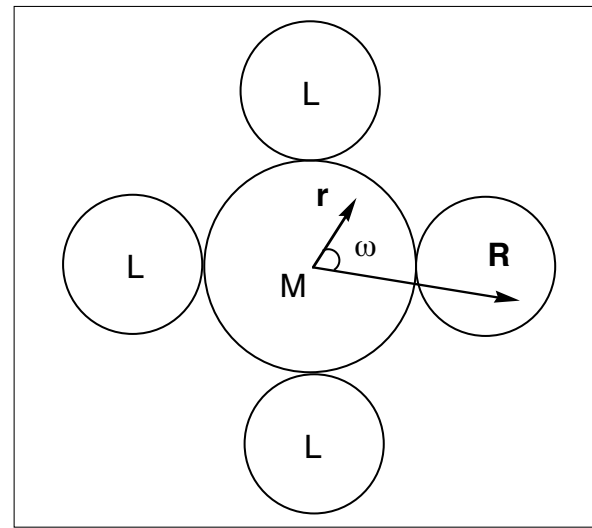

Fig. 1. Graphical representation of a metal center (M) surrounded by four ligands (L).

The term $1 /|\mathbf{r}-\mathbf{R}|$ in Eqn. (3) can be expanded in a Taylor series to obtain

$\frac{1}{|\mathbf{R}-\mathbf{r}|}=\frac{1}{r_{>}} \sum_{k=0}^{\infty}\left(\frac{r_{\leq}}{r_{>}}\right)^{k} P_{k}(\cos \omega)$

where, $\mathrm{r}_{<}$and $\mathrm{r}_{>}$are the lesser and the greater of the distances $\boldsymbol{r}$ and $\boldsymbol{R}$; and $\mathrm{P}_{\mathrm{k}}(\mathrm{x})$ is a Legendre polynomial of order $\mathrm{k}$. Without lack of generality we can assume that $r<$ $R$, i.e. considering formally that most of the density distributions is placed inside this specific region $(r<R)$. Therefore we write $\mathrm{r}_{<}=\mathrm{r}$ and $\mathrm{r}_{>}=\mathrm{R}$ and obtain the following (Eqn. (4)):

$$
\begin{aligned}
& \frac{1}{|\mathbf{R}-\mathbf{r}|}=\frac{1}{R} \sum_{k=0}^{\infty}\left(\frac{r}{R}\right)^{k} P_{k}(\cos \omega) \\
& =\frac{1}{R} \sum_{k=0}^{\infty} \frac{4 \pi}{2 k+1}\left(\frac{r}{R}\right)^{k} \sum_{m=-k}^{+k} Y_{k m}(\theta, \phi) Y_{k m}(\Theta, \Phi)
\end{aligned}
$$

where $Y_{l m}$ are spherical harmonics.
Thus $v_{L F}$ in Eqn. (3) can be rewritten (Eqn. (5)) and sometimes represented in the basis of the d-and/or the f-orbitals (Eqn. (6)) of the metallic center.

$$
v_{L F}(r, \theta, \phi)=\sum_{k=0}^{2 l} \sum_{q=-k}^{+k} h_{k q}(r) Y_{k q}(\theta, \phi)
$$

$$
\begin{aligned}
\left\langle l m\left|v_{L F}\right| l^{\prime} m^{\prime}\right\rangle & \\
& =\sum_{k=0}^{2 \max \left(l, l^{\prime}\right)} \sum_{q=-k}^{k} a_{k q}\left\langle l m\left|Y_{k q}\right| l^{\prime} m^{\prime}\right\rangle
\end{aligned}
$$

where, $a_{k q}=\left\langle h_{k q}\right\rangle_{\mathrm{R}}$ are adjustable parameters in the conventional ligand field theory and in the non-empirical LFDFT (vide infra). They describe the ligand field interaction. The integration $\left\langle\operatorname{lm}\left|Y_{k q}\right| l ' m\right.$ '> are simple coupling coefficients, i.e. $<l m\left|Y_{k q}\right| l^{\prime} m$ '> $=\Omega d \Omega Y_{l m}(\Omega) Y_{k q}(\Omega) Y_{l^{\prime} m},(\Omega)$. They can easily be evaluated by numerical quadrature.

Aiming to provide a practical insight to those ligand field parameters: $h_{k q}(r)$ (Eqn. (5)) and $a_{k q}$ (Eqn. (6)), it is useful to separate the influence of the ligand field interaction into two distinct sets: primo $h_{00}(r)$ and $a_{00}$ which denote the spherically symmetry field; and secondo $h_{k q}(r)$ and $a_{k q}$ with $\mathrm{k}>0$.

The <nll orbitals in an isolated gaseous metal are $(21+1)$-times degenerate. If a spherically symmetric field of negative charges involving expressions of $h_{o 0}(r)$ in Eqn. (5) and $a_{o 0}$ in Eqn. (6), is placed around the metal, these $<$ nll orbitals remain degenerate, but all of them are raised in energy as a result of the repulsion between the negative charges on the ligands and in the 1-orbitals on the metal ion. This phenomenon is known to be the nephelauxetic effect. Its consequences are depicted in Fig. 2.

Considering now the presence of an

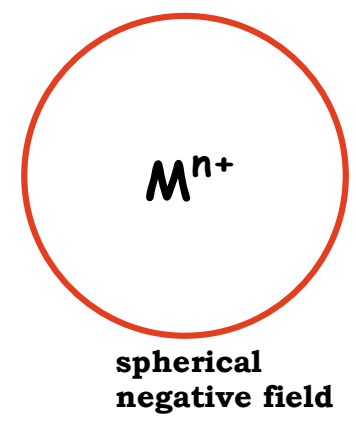

(a)

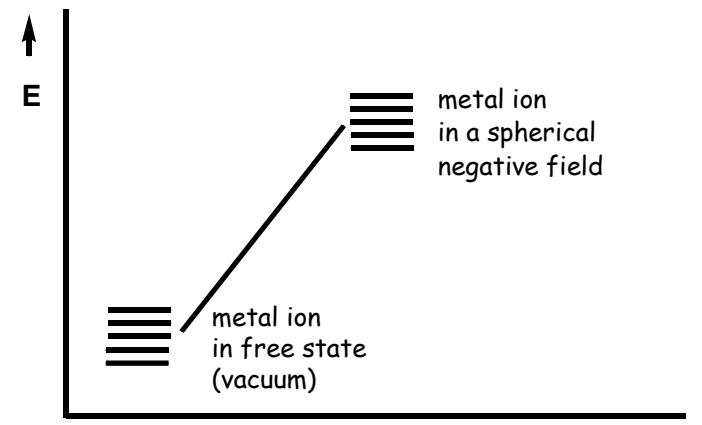

(b)

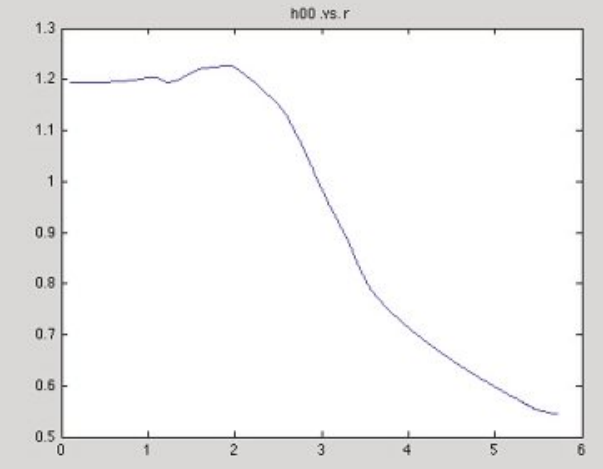

(c)

Fig. 2. Representations of: a metal ion surrounded by a spherical negative field (a); the influence of the spherical negative field onto the metal d orbitals (b); and parameters $h_{00}$ (in $10^{3} \mathrm{~cm}^{-1}$ ) with respect to $r$ (in $\AA$ ). 
octahedral ligand field for instance, i.e. six ligands are located on the vertices of an octahedron $(+\mathrm{x},-\mathrm{x},+\mathrm{y},-\mathrm{y},+\mathrm{z}$ and $-\mathrm{z}$ axes respectively) and allowed to interact onto the metal center, the degeneracy of the $\mathrm{d}$ orbitals is removed governed by simple group theory rule. The expression of the spherically symmetric field (Fig. 2) is no longer sufficient and the $\mathrm{d}$ orbitals which are lying along the $\mathrm{x}, \mathrm{y}$ and $\mathrm{z}$ axes (i.e. $\mathrm{d}_{\mathrm{x} 2-\mathrm{y} 2}$ and $\mathrm{d}_{22}$ orbitals) will be destabilized more than the orbitals which are lying inbetween the axes (i.e. $\mathrm{d}_{\mathrm{xy}}, \mathrm{d}_{\mathrm{xz}}, \mathrm{d}_{\mathrm{yz}}$ ). In Fig. 3 we show the full action of an octahedral ligand field. The ligand field potential in the case of an octahedral complex is the following:

$$
\begin{aligned}
V_{O_{h}}=- & \frac{3}{2} \Delta_{o} Y_{4-4}-\frac{5}{2} \Delta_{o} Y_{40} \\
& -\frac{3}{2} \Delta_{o} Y_{44}+\operatorname{tr}\left(\left\langle\ell m\left|V_{O_{h}}\right| \ell m^{\prime}\right\rangle\right)
\end{aligned}
$$

where, only one parameter prevails i.e. $\Delta_{0}$ the energy difference between the $e_{\mathrm{g}}\left(\mathrm{d}_{\mathrm{x} 2-\mathrm{y} 2}\right.$ and $\mathrm{d}_{\mathrm{z} 2}$ orbitals $)$ and $\mathrm{t}_{2 \mathrm{~g}}\left(\mathrm{~d}_{\mathrm{xy}}, \mathrm{d}_{\mathrm{xz}}, \mathrm{d}_{\mathrm{yz}}\right)$ orbitals (cf. Fig. 3).

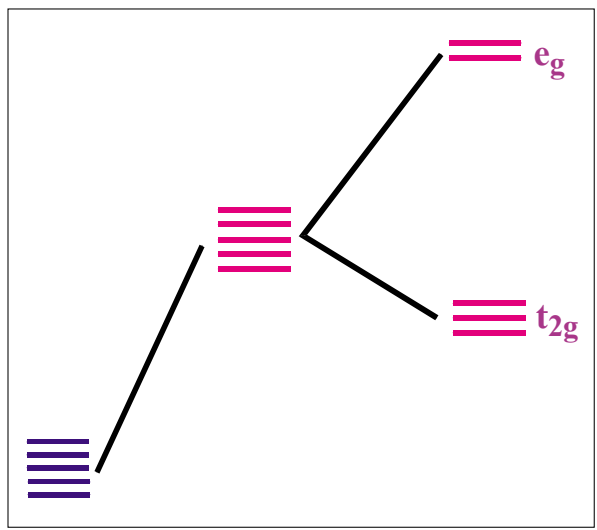

Fig. 3. Splitting of the d orbitals according to an octahedral ligand field.

Before getting straight to the topic of interest, i.e. ligand field theory, the prediction of the electronic fine structure of openshell systems requires first the wave function of the free metal ion. A simple method to solve the atomic Kohn-Sham equation is proposed, the Matlab codes (XATOM) are available from the author upon request.

\section{Method to Solve the Atomic Kohn- Sham Equation Numerically}

For a free metallic ion, the radial KohnSham wave functions can be written in the following form (Eqn. 8):

$$
\begin{array}{r}
{\left[-\frac{d^{2}}{d r^{2}}+\frac{\lambda(\lambda+1)}{r^{2}}+V(r)\right]} \\
P_{n \lambda}(r)=E_{n \lambda} P_{n \lambda}(r)
\end{array}
$$

where $P_{n \lambda}(r)=r R_{n \lambda}(r)$ are normalized radial wave functions, i.e.

$$
\int_{0}^{\infty}\left[P_{n \lambda}(r)\right]^{2} d r=1
$$

and $V(r)$ is the sum of the nuclear coulomb potential, the total electronic coulomb potential, and the exchange potential. One will tentatively set $V(r)$ equal to $\mathrm{V}_{0}(\mathrm{r})$ at all values of $\mathrm{r}$, where $\mathrm{V}_{0}(\mathrm{r})$ is defined as follows (Eqn. (9)):

$$
\begin{aligned}
\mathrm{V}_{0}(\mathrm{r})=- & \frac{2 \mathrm{Z}}{\mathrm{r}}+\left(\frac{2}{\mathrm{r}}\right) \int_{0}^{\mathrm{r}} \sigma(\mathrm{t}) \mathrm{dt} \\
& +2 \int_{\mathrm{r}}^{\infty} \frac{\sigma(\mathrm{t})}{\mathrm{t}} \mathrm{dt}-6\left[-\frac{3}{8 \pi} \rho(\mathrm{r})\right]^{1 / 3}
\end{aligned}
$$

where $\mathrm{Z}$ is the atomic number; and

$\rho(r)=\frac{\sigma(r)}{4 \pi r^{2}}$

is the spherically averaged total electronic charge density (both spins). It is noteworthy to highlight that the electronic coulomb potential is expressed in terms of $\sigma(r)$, which is the total electronic charge density $\left(\sigma(\mathrm{r})=\sum_{\mathrm{n} \lambda} \omega_{\mathrm{n} \lambda}\left[\mathrm{P}_{\mathrm{n} \lambda}(\mathrm{r})\right]^{2}\right)$;

and the free-electron exchange potential in terms of $\rho(r)$. Both distances and energies are measured in atomic unit, i.e. in Bohr units and in Rydberg units, respectively. $\omega_{n \lambda}$ is the occupation number for the orbital $n \lambda$ (both spins). In the special case of a closed shell, $\omega_{n \lambda}=2(2 \lambda+1)$. More generally, the total number of electrons and the ionicity can be obtained from Eqns. (10) and (11), respectively.

$$
N=\sum_{n \lambda} \omega_{n \lambda}
$$

$$
\mathrm{q}_{\text {ion }}=\mathrm{Z}-\mathrm{N}
$$

Unfortunately, the free-electron approximation breaks down at large distances from the nucleus. This observation has been made more than half of a century ago by R. Latter. ${ }^{[10]}$ His essential idea was to redefine V(r) so that it has the correct asymptotic behavior at large $r$. Let us consider an atom or ion with atomic number $\mathrm{Z}$ containing $\mathrm{N}$ electrons. Let the above-defined potential be denoted by $\mathrm{V}_{0}(\mathrm{r})$, and let us call this the unmodified Kohn-Sham potential. Let us denote by $\mathrm{V}(\mathrm{r})$ the potential that is actually introduced into the wave equation and let us call this the tail-corrected KohnSham potential. We shall now define V(r) in terms of $\mathrm{V}_{0}(\mathrm{r})$ as follows (Eqn. (12))

$$
V(r)= \begin{cases}V_{0}(r) & \text { if } r<r_{0} \\ -\frac{2(Z-N+1)}{r} & \text { if } r \geq r_{0}\end{cases}
$$

where $r_{0}$ is the value of $r$ at which

$V_{0}(r)=-\frac{2(Z-N+1)}{r}$,

i.e. calculated using Eqn. (13):

$$
V_{0}\left(r_{0}\right)=-\frac{2(Z-N+1)}{r_{0}}
$$

Thus, the free-electron exchange approximation is used only in the interior region, where it is satisfactory, and the self-coulomb potential is properly taken into account at large values of $r$. The behavior of $\mathrm{V}(\mathrm{r})$ at intermediate $r$ is of course subject to question, and the discontinuous behavior of

$$
\left(\frac{d V}{d r}\right)_{r=r_{0}}
$$

is distinctly 'unphysical'. In spite of its arbitrary character in the neighborhood of $r=$ $\mathrm{r}_{0}$, the modified potential $\mathrm{V}(\mathrm{r})$ is decidedly better than the unmodified potential $\mathrm{V}_{0}(\mathrm{r})$.

\section{Numerical Solution of the Radial Wave Equation}

The Eigen solutions of the Kohn-Sham radial wave equation (Eqn. (8)) are obtained using the following procedure. The radial wave function $P_{n \lambda}(r)$ must be 0 not only at $\mathrm{r}=0$ but also at $\mathrm{r}=\infty$. In the range $0<\mathrm{r}<\infty, P_{n \lambda}(r)$ must possess exactly $\mathrm{n}-\lambda-1$ nodes.

Using finite difference approximation for $\frac{d^{2} f(r)}{d r^{2}}$

we easily get (Eqn. (14))

$$
\begin{gathered}
\left(\frac{d^{2} f}{d r^{2}}\right)_{r=r_{i}} \approx \frac{2 f\left(r_{i-1}\right)}{\left(r_{i}-r_{i-1}\right)\left(r_{i+1}-r_{i-1}\right)} \\
-\frac{2 f\left(r_{i}\right)}{\left(r_{i}-r_{i-1}\right)\left(r_{i+1}-r_{i-1}\right)}-\frac{2 f\left(r_{i}\right)}{\left(r_{i+1}-r_{i}\right)\left(r_{i+1}-r_{i-1}\right)} \\
+\frac{2 f\left(r_{i+1}\right)}{\left(r_{i+1}-r_{i}\right)\left(r_{i+1}-r_{i-1}\right)}+\varepsilon\left(\delta r^{3}\right)
\end{gathered}
$$

where $r_{i}$ are points on the radial grid. A convenient choice for the radial mesh is given by $r_{k}=c\left(e^{k h}-1\right)$ where $h=0.14$, $\mathrm{c}=0.000852$ and $\mathrm{k}_{\max }=125$. These values were simply obtained by trial and error. The Matlab script XATOM allows to perform the calculation. To illustrate this, Table 1 and Fig. 4 show the results of an XATOM calculation for a lanthanide $\mathrm{Gd}^{3+}$ for example. 


$$
\left\langle\Phi_{\mu}|G| \Phi_{v}\right\rangle=\left\{\begin{array}{c}
0 \quad \text { if } \Phi_{\mu} \neq \Phi_{v} \text { by more than two spinorbitals } \\
\pm\left[\left\langle\chi_{k} \chi_{m}|g| \chi_{l} \chi_{n}\right\rangle-\left\langle\chi_{k} \chi_{l}|g| \chi_{m} \chi_{n}\right\rangle\right] \text { if } \Phi_{\mu} \neq \Phi_{v} \text { by exactly } 2 \text { spinorbitals }\left\{\chi_{k} \chi_{l}\right\} \text { and }\left\{\chi_{m} \chi_{n}\right\} \\
\pm \sum_{t}\left[\left\langle\chi_{k} \chi_{t}|g| \chi_{t} \chi_{t}\right\rangle-\left\langle\chi_{k} \chi_{t}|g| \chi_{t} \chi_{l}\right\rangle\right] \text { if } \Phi_{\mu} \neq \Phi_{v} \text { by exactly } 1 \text { spinorbital } \chi_{k} \text { and } \chi_{l} \\
\sum_{k>t}\left[\left\langle\chi_{k} \chi_{k}|g| \chi_{t} \chi_{t}\right\rangle-\left\langle\chi_{k} \chi_{t}|g| \chi_{k} \chi_{t}\right\rangle\right] \text { if } \Phi_{\mu}=\Phi_{v}
\end{array}\right.
$$

Table 1. XATOM calculation for $\mathrm{Gd}^{3+}$

Output: Energies are given in Rydberg units. converged

$\begin{array}{ll}\text { ekin } & =21608 \\ \text { enuc } & =-51386 \\ \text { ecoul } & =8511.8 \\ \text { exc } & =-451.36 \\ \text { etot } & =-21718\end{array}$

s-orbitals (energy versus occupation)

$\begin{array}{ll}-3464.9 & 2 \\ -552.22 & 2 \\ -122.37 & 2 \\ -\quad 24.37 & 2 \\ -\quad 3.2522 & 2 \\ -\quad 0.32733 & 2\end{array}$

p-orbitals (energy versus occupation)

$-520.84 \quad 6$

$-109.03 \quad 6$

$\begin{array}{ll}- & 19.265 \\ -\end{array}$

- $1.8872 \quad 6$

d-orbitals (energy versus occupation)

$\begin{array}{ll}-85.598 & 10\end{array}$

$\begin{array}{ll}- & 10.584 \quad 10\end{array}$

f-orbitals (energy versus occupation)

- 0.48387

Next we describe the calculation of the electrostatic multiplets.

\section{Multiplet Calculation}

The multiplet wave $\psi_{\mathrm{i}}$ that belongs to a given configuration $\alpha$ arising from an electron configuration $1^{\mathrm{n}}\left(\psi_{\mathrm{i}}=\mid \alpha L M_{L} S\right.$ $\left.\left.M_{S}\right\rangle\right)$ are obtained by solving the secular equation $\mathbf{H ~ C ~}=\mathbf{C} \mathbf{E}$ taking as basis all the Slater-determinants in the model space, i.e.

$$
\Psi_{i}=\sum_{\mu} C_{i \mu} \Phi_{\mu}
$$

$L$ is the label of the angular momentum (irreducible representation, irreps) of the space part of the wave function; $M_{L}$ refers to its component in case of degeneracy, $S$ is the spin part of the wave function with component $\left.M_{S} ; \mathrm{H}_{\mu v}=<\mathrm{F}_{\mu}\left|\mathrm{H}_{0}+\mathrm{G}_{12}\right| \mathrm{F}_{v}\right\rangle ; \Phi_{\mu}$ $=\mid \chi_{1} \chi_{2} \chi_{3} \ldots$ is a $\mathrm{N}$-electron wave function of spin-orbitals $\chi_{1}, \chi_{2}, \chi_{3}, \ldots$; and $\mathrm{C}_{\mathrm{i} u}$ is an orthogonal square matrix of the Eigenvectors. For a particular $\chi_{1}$ we have $\chi_{1}=\left|l m_{l} s m_{s}\right\rangle_{k}$. This task is best achieved automatically in a computer program. The procedure is briefly described next.

The matrix elements $\mathrm{H}_{u v}$ can be easily obtained from Slater's rules for a single determinant as a sum of coulomb and exchange integrals (ignoring the constant one-electron part for the determinants of a single configuration originating from $\mathrm{H}_{0}$ ) as given in Eqn. (15) where $\mathrm{G}=1 / \mathrm{r}_{1}$.

Thus we are finally left with the task to evaluate the one-center two-electron electrostatic matrix elements $<\chi_{\mathrm{a}} \chi_{\mathrm{b}}|\mathbf{g}| \chi_{\mathrm{c}} \chi_{\mathrm{d}}>$ $=<1 \mathrm{~m}_{\mathrm{a}} \mathrm{l}_{\mathrm{b}} \mathrm{m}|\mathbf{g}| \mathrm{l}_{\mathrm{c}} \mathrm{m}_{\mathrm{c}} \mathrm{l}_{\mathrm{d}}>\delta_{\mathrm{d}, \mathrm{c}} \delta_{\text {sb sd }}$ since there is no spin contribution to the electrostatic repulsion. Moreover, the electrostatic repulsion operator is most conveniently written as Eqn. (16) where like in Eqn. (4), $r_{<}$and $r_{>}$become the lesser and the greater of the distances $r_{1}$ and $r_{2}$ of two electrons from the nuclei.

Considering a specific two-electron integral $\left\langle l_{a} m_{a}(1) l_{b} m_{b}(2)|g| l_{c} m_{c}(1) l_{d} m_{d}(2)\right\rangle$, where $m_{a}, m_{b}, m_{c}$ and $m_{d}$ denote the corresponding components of a $l_{a}$-orbital or a $l_{b}$-orbital etc. (Eqn. (17), Eqn. (18)).

The parameters $\mathrm{W}_{\mathrm{k}}$ are the radial integral of the atomic wave functions. They are

$$
\frac{1}{r_{12}}=\sum_{k} \frac{2 \pi}{2 k+1} \frac{r_{<}^{L}}{r_{>}^{L+1}} \sum_{m=-k}^{m=k} Y_{k m}\left(\theta_{1}, \phi_{1}\right) Y_{k m}^{*}\left(\theta_{2}, \phi_{2}\right)=\sum_{k m} g_{k, m}^{(1)} g_{k, m}^{(2)}
$$

$$
\begin{aligned}
& \left\langle\ell_{a} m_{a}(1) \ell_{b} m_{b}(2)|g| \ell_{c} m_{c}(1) \ell_{d} m_{d}(2)\right\rangle \\
& =\sum_{k} \frac{4 \pi}{2 k+1}\left\langle R_{n_{a} l_{a}} R_{n_{b} l_{b}}\left|\frac{r_{<}^{k}}{r_{>}^{k+1}}\right| R_{n_{c} l_{c}} R_{n_{d} l_{d}}\right\rangle \sum_{m=-k}^{m=k}\left(\begin{array}{ccc}
l_{a} & k & l_{c} \\
m_{a} & m & m_{c}
\end{array}\right)\left(\begin{array}{ccc}
l_{b} & k & l_{d} \\
m_{b} & m & m_{d}
\end{array}\right) \\
& =\sum_{k} \frac{4 \pi}{2 k+1} W_{k}\left(n_{a} l_{a}, n_{b} l_{b}, n_{c} l_{c}, n_{d} l_{d}\right) A_{k}\left(l_{a} m_{a}, l_{b} m_{b}, l_{c} m_{c}, l_{d} m_{d}\right)
\end{aligned}
$$

$$
\begin{aligned}
& W_{k}\left(n_{a} l_{a}, n_{b} l_{b}, n_{c} l_{c}, n_{d} l_{d}\right)=\int \frac{r_{<}^{k}}{r_{>}^{k+1}} R_{n_{a} l_{a}}(1) R_{n_{b} l_{b}}(2) R_{n_{c} l_{c}}(1) R_{n_{d} l_{d}}(2) r_{1}^{2} r_{2}^{2} d r_{1} d r_{2} \\
& A_{k}\left(l_{a} m_{a}, l_{b} m_{b}, l_{c} m_{c}, l_{a} m_{d}\right)=\sum_{m=-k}^{m=k}\left(\begin{array}{ccc}
l_{a} & k & l_{c} \\
m_{a} & m & m_{c}
\end{array}\right)\left(\begin{array}{ccc}
l_{b} & k & l_{d} \\
m_{b} & m & m_{d}
\end{array}\right)
\end{aligned}
$$

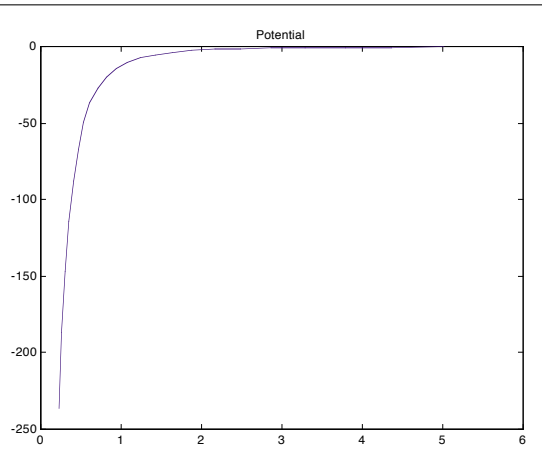

(a)

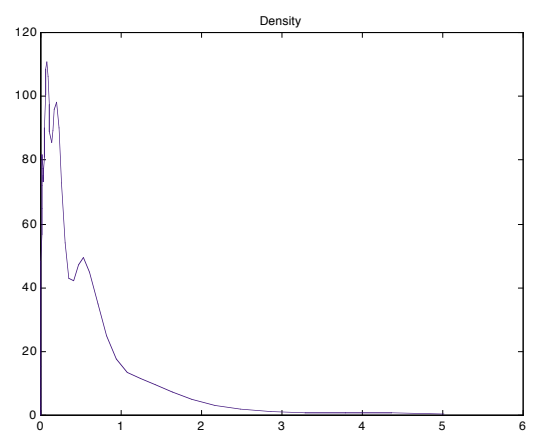

(b)

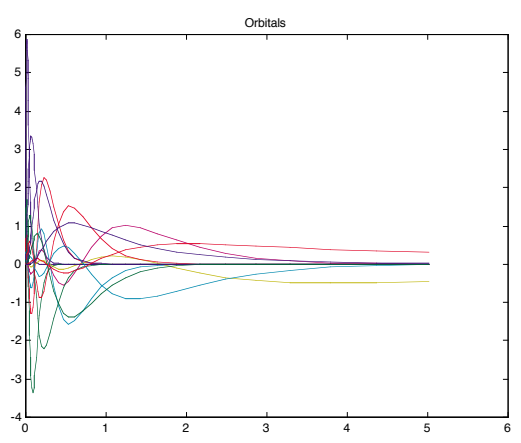

(c)

Fig. 4. Graphical representations of the potential (a); the density (b); and the radial wave functions $P_{n \lambda}$ (c) obtained by X-atom calculation of lanthanide $\mathrm{Gd}^{3+}$. 


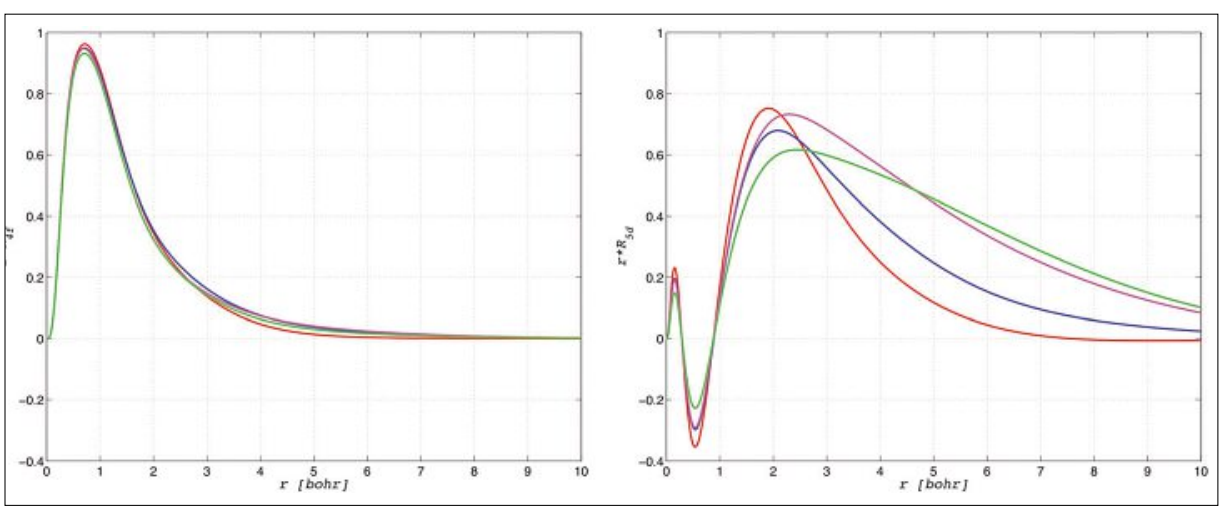

Fig. 5. Graphical representation of the radial functions of the $4 \mathrm{f}$ (left hand side) and $5 \mathrm{~d}$ (right hand side) Kohn-Sham orbitals of the free $\mathrm{Pr}^{3+}$ ion (in red) and the complexes $\left(\mathrm{PrF}_{8}\right)^{5-}$ (in blue), $\left(\mathrm{PrCl}_{8}\right)^{5-}$ (in magenta) and $\left(\mathrm{PrBr}_{8}\right)^{5-}$ (in green), forming a $\mathrm{D}_{4 \mathrm{~h}}$ arrangement.

rigorously known as the Condon-Shortley radial parameters or simply the SlaterCondon or Racah parameters and can be adjusted to experimental data or calculated from first principle (Eqn. (18)). In this latter case, it is essential to use radial functions that are properly corrected for the nephelauxetic effect afore-mentioned. In Fig. 5 , one can illustrate the expansion of the radial $5 \mathrm{~d}$ and $4 \mathrm{f}$ functions for various $\mathrm{Pr}$ halides complexes for instance. The $\mathrm{A}_{\mathrm{k}}$ 's are Gaunt integrals and are just numbers that are easily calculated. In fact very few $\mathrm{A}_{\mathrm{k}}$ 's are non-zero since the triangle condition and the even lower index rule must apply for both Clebsch-Gordan coefficients (Eqn. (18)). The electrostatic two-electron integral < ablcd $>$ will vanish if there is no $\mathrm{k}$ value for which these conditions are met.

\section{Effective Ligand Field Hamiltonian}

Following the effective Hamiltonian approach, let us consider the KS orbitals dominated by $l$-functions, which result from an AOC $l^{\mathrm{n}}$ DFT-SCF calculation. This yields $2 l+1$ eigenvalues $\varepsilon_{\mathrm{i}}$ and eigenvectors $\vec{U}_{i}$. From the components of this eigenvector matrix built up from such MOs one takes only the components corresponding to the $l$-functions. Let us denote this $(2 l+1) \times(2 l+1)$ square matrix composed of the new column vectors by $\mathbf{U}$ and introduce the overlap matrix $\mathbf{S}$ :

$$
\mathbf{S}=\mathbf{U U}^{\mathrm{T}}
$$

Since $\mathbf{U}$ is in general not orthogonal, we use Löwdin's symmetric orthogonalization procedure to obtain an equivalent set of orthogonal eigenvectors $(\mathbf{c})$ :

$$
\mathbf{c}=\mathbf{S}^{\frac{1}{2}} \mathbf{U}
$$

We identify now these vectors as the Eigenfunctions of the effective LF Hamiltonian $\widehat{h}_{L F}^{\text {eff }}$ sought, as

$$
\phi_{i}=\sum_{\mu=1}^{2 \ell+1} c_{\mu i}\left|\ell_{\mu}\right\rangle
$$

This procedure will enable us to estimate the full representation matrix of $\widehat{h}_{L F}^{\text {eff }}$ (Eqn. (22)) as

$$
h_{\mu v}=\left\langle\ell_{\mu}\left|\hat{h}_{L F}^{e f f}\right| \ell_{v}\right\rangle=\sum_{\mu=1}^{2 \ell+1} c_{\mu i} \varepsilon_{i} c_{v i}
$$

\section{Relativistic Corrections: The ZORA Equation for Atoms}

The spin-orbit splitting is already present in the zero ${ }^{\text {th }}$ order Hamiltonian. This spin-orbit term is regular because of the $\left(2 \mathrm{c}^{2}-\mathrm{V}\right)^{-2}$ factor in it. It poses no problem in variational calculations. The Eigenvalue equation:

$$
H^{\text {zora }} \boldsymbol{\Phi}^{\text {zora }}
$$

$$
=\left(V+\vec{\sigma} \cdot \vec{p} \frac{c^{2}}{2 c^{2}-V} \vec{\sigma} \cdot \vec{p}\right) \Phi^{z o r a}=E^{z o r a} \Phi^{z o r a}
$$

is only a second-order differential equation (cf. Eqn. (22)). The two-component wave function $\Phi^{\text {zora }}$ will now be referred to as the ZORA wave function.

\section{SCF Calculations}

We discuss first the construction of the potential $\mathrm{V}(\mathrm{r})$ in the Hamiltonian $\mathrm{H}^{\text {zora }}$ from the one-electron solutions to Dirac's equation above during the iterations of a SCF calculation. In the present work the simple $\mathrm{X} \alpha$ version of DFT is used for the sake of simplicity. The electron-electron potential $\mathrm{V}_{\mathrm{ee}}$ is thus split in the classical Coulomb interaction $\mathrm{V}_{\mathrm{C}}$ and the exchange-correlation potential $\mathrm{V}_{\mathrm{xc}}$. Magnetic effects and retardation are not taken into account for the electron-electron potential. The potentials are calculated from the electron density $\rho$ in the following way (Eqn. (24)):
$\mathrm{V}=\mathrm{V}_{\mathrm{N}}+\mathrm{V}_{\mathrm{ee}}=\mathrm{V}_{\mathrm{N}}+\mathrm{V}_{\mathrm{C}}+\mathrm{V}_{\mathrm{xc}}$

$c f$. Eqn. (25) where the different terms are explicitly given.

$$
\begin{aligned}
& V_{N}(\vec{r})=-\frac{Z}{r} \\
& V_{C}(\vec{r})=\int \frac{\rho\left(\vec{r}_{2}\right)}{\left|\vec{r}-\vec{r}_{2}\right|} d \vec{r}_{2} \\
& V_{X C}(\vec{r})=V_{X C}(\rho)=-3 \alpha\left[\frac{3}{8 \pi} \rho(\vec{r})\right]^{\frac{1}{3}}
\end{aligned}
$$

with $\alpha=0.7$. The Dirac equation with this approximation for the exchange-correlation potential is called the Dirac-Slater equation.

\section{Separation of the Radial Variable from Angular and Spin Variables}

To solve the equations for an atom it is useful to separate variables just like in the non-relativistic case. Here we follow the standard approach, which can be found in any text book on relativistic effects. Because the potential of an atom is spherically symmetric, the total angular momentum $\vec{j}=\vec{l}+\vec{s}$ of a particle is conserved. $\vec{j}$ commutes with the ZORA Hamiltonian, so we may construct simultaneous Eigenfunctions of $\mathrm{H}^{\text {zora }}, \mathrm{j}^{2}$ and $\mathrm{j}_{\mathrm{z}}$. The Eigenfunctions can also be classified according to parity. It is convenient to introduce the operator (Eqn. (26))

$$
\hat{\kappa}=\vec{\sigma} \cdot \vec{l}+1=\vec{\sigma} \cdot(\vec{r} \times \vec{p})+1
$$

Eigenfunctions of this operator are written as $\eta_{\kappa}^{m}$ with eigenvalue $-\kappa($ Eqn. (27))

$$
\hat{\kappa} \eta_{\kappa}^{m}=-\kappa \eta_{\kappa}^{m}
$$

These are functions of angular and spin variables with a definite parity. The relativistic quantum number $\kappa$ is given (Eqn. (28)) by:

$$
\kappa= \begin{cases}-(l+1)=-\left(j+\frac{1}{2}\right) & j=l+\frac{1}{2} \\ +l=+\left(j+\frac{1}{2}\right) & j=l-\frac{1}{2}\end{cases}
$$

$\eta_{\kappa}^{m}$ is explicitly given by (Eqn. (29))

$$
\eta_{\kappa}^{m}=\sum_{m^{\prime}, m_{s}}\left\langle l m^{\prime} s m_{s} \mid j m\right\rangle Y_{l m^{\prime}} \theta_{s m_{s}}
$$


In this equation $\mathrm{m}=\mathrm{m}$ ' $+\mathrm{m}$ holds and $\theta_{\text {sms }}$ is the Eigenfunction of $S^{2}$ and $S_{z}$. One can now separate variables. Writing $\Phi=R(r) \eta_{\kappa}^{m}$ it is possible to calculate $\vec{\sigma} \cdot \vec{p} \Phi$ (Eqns. (30) and (31)):

\section{$\sigma_{r} \equiv \frac{\vec{\sigma} \cdot \vec{r}}{r} ; \quad \sigma_{r} \eta_{\kappa}^{m}=-\eta_{\kappa}^{m} ; \quad \sigma_{r}^{2}=1 \quad(30)$ \\ Example 1: Calculation of the Magnetic Anisotropy of Single Ion Magnets}

$$
\begin{aligned}
\vec{\sigma} \cdot \vec{p} & \Phi=\sigma_{r}^{2} \vec{\sigma} \cdot \vec{p} \Phi=\frac{\vec{\sigma} \cdot \vec{r}}{r}\left(\frac{\vec{\sigma} \cdot \vec{r}}{r} \vec{\sigma} \cdot \vec{p}\right) \Phi \\
= & \sigma_{r}\left(\frac{1}{r} \vec{r} \cdot \vec{p}+\frac{1}{r} \vec{\sigma} \cdot(\vec{r} \times \vec{p})\right) \Phi \\
= & \sigma_{r}\left(-i \frac{\partial}{\partial r}+\frac{i}{r} \vec{\sigma} \cdot \vec{l}\right)\left(R(r) \eta_{\kappa}^{m}\right) \\
& =\sigma_{r}\left(-i \frac{\partial R}{\partial r}-i \frac{\kappa+1}{r} R\right) \eta_{\kappa}^{m} \\
& =i\left(\frac{\partial R}{\partial r}+\frac{\kappa+1}{r} R\right) \eta_{-\kappa}^{m}
\end{aligned}
$$

Now it is possible to separate the spin and angular variables from the radial variable in the ZORA-Slater equations, because the $\vec{\sigma} \cdot \vec{p}$ operator appears twice. The first one will give something proportional to $\eta_{-\kappa}^{m}$ the second one will give back something proportional to $\eta_{\kappa}^{m}$.

Due to the separation of radial from angular and spin variables, the Dirac-Slater and the ZORA-Slater equation can be solved numerically. This will be done for the d- and/or f-elements using our atomic Kohn-Sham solver.

To solve the ZORA-Slater equation for atoms, one needs to calculate the following matrix elements:

$$
\begin{aligned}
\left\langle\Phi_{i} \mid \Phi_{j}\right\rangle ; & \left\langle\Phi_{i}|V| \Phi_{j}\right\rangle \\
& \frac{1}{2}\left\langle\Phi_{i}|\vec{\sigma} \cdot \vec{p} f \vec{\sigma} \cdot \vec{p}| \Phi_{j}\right\rangle
\end{aligned}
$$

where $\Phi_{i}=R_{i}(r) \eta_{\kappa_{i}}^{m_{i}} ; \quad f=\frac{1}{1-V / 2 c^{2}}$

The first two are the same as in the nonrelativistic case. The last one can be written as (Eqn. (32)):

$$
\begin{aligned}
& \frac{1}{2}\left\langle\Phi_{i}|\vec{\sigma} \cdot \vec{p} f \vec{\sigma} \cdot \vec{p}| \Phi_{j}\right\rangle=\frac{1}{2} \delta_{m_{i} m_{j}} \delta_{\kappa_{i} \kappa_{j}} \\
& \times \int_{0}^{\infty}\left(\frac{\partial R_{i}}{\partial r}+\frac{\kappa+1}{r} R_{i}\right) \cdot\left(\frac{\partial R_{j}}{\partial r}+\frac{\kappa+1}{r} R_{j}\right) f r^{2} d r
\end{aligned}
$$

Because $\mathrm{f}=\left(1-\mathrm{V} / 2 \mathrm{c}^{2}\right)^{-1}$ depends on the potential, it is convenient to do a numerical integration. In order to solve the ZORA-Slater equations, one can use the same techniques as in the non-relativistic case $e . g$. in XATOM. Table 2 illustrates the relation between $\kappa$ and $|l j\rangle$

Table 2. $\kappa$-Blocks

\section{$\mathrm{s}_{1 / 2}-\mathrm{k}=-1$ $\begin{array}{ll}\mathrm{p}_{1 / 2} & \kappa=1 \\ \mathrm{p}_{3 / 2} & \kappa=-2\end{array}$} theoretical point-of-view.

Table 3. a) M.O. energies of the metallic $f-$ orbitals; b) Eigenvectors corresponding to the Eigenvalues in Table $3 a$.

a)

b)
It has long been recognized that metal spin states play a central role the new technologies, in the reactivity of important biomolecules, in industrial catalysis and in spin crossover compounds. The latter offer many exciting possibilities for novel, switchable materials with applications in computer storage and display devices. Elucidating the role and effect of different spin states on the properties of a system is presently one of the most challenging endeavors both from an experimental and

Considering tetraphenylarsonium pentakis(nitrato)ytterbate(III) ${ }^{[11]}$ as a prototype for Single Ion Magnets (SIMs), we present methodological advances and state-of-the art computations analyzing the electronic structure and its relationship with the magnetic properties due to the

\begin{tabular}{|c|c|c|c|c|c|c|c|}
\hline & $4 b$ & $3 b$ & $3 a$ & $2 b$ & $2 \mathrm{a}$ & $1 b$ & $1 \mathrm{a}$ \\
\hline $4 f-3$ & 0.0000 & 0.0000 & 0.1917 & 0.0000 & -0.7869 & -0.0000 & -0.5633 \\
\hline $4 f-2$ & -0.0000 & 0.0000 & 0.5759 & 0.0000 & -0.3509 & 0.0000 & 0.7183 \\
\hline $4 f-1$ & 0.0000 & 0.0000 & 0.7550 & -0.0000 & 0.4683 & -0.0000 & -0.4060 \\
\hline 4f_0 & -0.0022 & 0.8522 & -0.0000 & -0.2875 & -0.0000 & 0.3276 & 0.0000 \\
\hline $4 f+1$ & 0.3047 & 0.1399 & -0.0000 & -0.4487 & -0.0000 & -0.8109 & 0.0000 \\
\hline $4 f+2$ & -0.5479 & -0.2759 & 0.0000 & -0.7245 & -0.0000 & 0.1241 & -0.0000 \\
\hline $4 f+3$ & 0.6851 & -0.2842 & 0.0000 & -0.3844 & -0.0000 & 0.4628 & -0.0000 \\
\hline
\end{tabular}
$\mathrm{Yb}(\mathrm{III})$ ion. Fig. 6 represents its structure.

The results of the quantum chemical calculations are quantitatively decrypted in the framework of Ligand Field (LF) theory, extracting the full parametric sets and interpreting in heuristic key the outcome. An important result is the characterization

\begin{tabular}{|l|l|l|}
\hline MO\# & energy & occ \\
\hline 30 & -13.743 & 1 \\
\hline 31 & -13.780 & 2 \\
\hline 32 & -13.810 & 2 \\
\hline 33 & -13.821 & 2 \\
\hline 34 & -13.825 & 2 \\
\hline 35 & -13.853 & 2 \\
\hline 36 & -13.855 & 2 \\
\hline
\end{tabular}

\begin{tabular}{ll|ll}
$\mathrm{d}_{3 / 2}$ & $\kappa=2$ & $\mathrm{f}_{5 / 2}$ & $\kappa=3$ \\
$\mathrm{~d}_{5 / 2}$ & $\kappa=-3$ & $\mathrm{f}_{7 / 2}$ & $\kappa=-4$
\end{tabular}

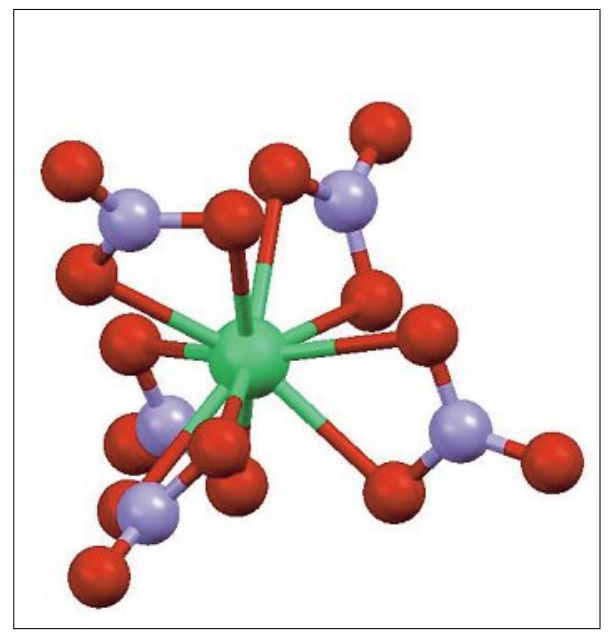

Fig. 6. Structure of $\mathrm{Yb}\left(\mathrm{NO}_{3}\right)_{5}^{-}$(color code: $\mathrm{Yb}$ in green, $\mathrm{N}$ in blue and $\mathrm{O}$ in red).

of the UV-Vis spectra and of the magnetic properties in the ground and excited states. We use the methodology described above. The ground state is characterized by a large anisotropy of the g-tensor and the excited states by the optical spectra. The predicted and observed findings are in excellent agreement.

\section{Description of our Results}

First we perform a DFT calculation of the above-mentioned molecule by occupying the M.O. with dominant f-character equally, i.e. 13/7 in each. The M.O. energies and the corresponding Eigenvectors thus obtained are listed in Table 3 (only the dominant metallic contribution is given):

Next, we use the effective Hamiltonian method to calculate the LF-matrix (Table 4 ; units are $10^{3} \mathrm{~cm}^{-1}$ ):

And in Table 5 we give the equivalent multipolar representation of the LF:

A ZORA calculation yields the spin orbit coupling constant $\zeta_{4 \mathrm{f}}$ (ZORA $=$ 3'280 $\mathrm{cm}^{-1}$; the calculated orbital reduction factor is $\mathrm{k}_{\text {orb }}=0.908$, thus $\zeta_{4 \mathrm{f}}=0.91 * 3$ '280 $=2^{\prime} 980 \mathrm{~cm}^{-1}$. Using this spin-orbit cou- 
Table 4. Ligand-Field matrix of $\mathrm{Yb}\left(\mathrm{NO}_{3}\right)_{5}^{-}$

\begin{tabular}{|c|c|c|c|c|c|c|c|}
\hline \multirow[t]{7}{*}{$\left\langle f_{m}\left|\mathrm{~V}_{L F}\right| f_{m}\right\rangle=$} & {$[0.1700$; } & & & & & & \\
\hline & 0.1123 & 0.1596; & & & & & \\
\hline & -0.0367 ; & 0.1268 & 0.2755 & & & & \\
\hline & 0 & 0 & 0 & 0.5126 & & & \\
\hline & 0 & 0 & 0 & 0.1126 & 0.1787; & & \\
\hline & 0 & 0 & 0 & -0.0963 & -0.1101 ; & 0.5184 & \\
\hline & 0 & 0 & 0 & -0.1284 & 0.2349 & -0.2605 ; & $0.5892]$ \\
\hline
\end{tabular}

Table 5. Multipolar representation of the Ligand Field of $\mathrm{Yb}\left(\mathrm{NO}_{3}\right)_{5}^{-}$(term of $\mathrm{a}_{\mathrm{kq}}$ cf. Eqn. (6)).

$\mathrm{a}_{00}=0$

$\mathrm{a}_{2 \mathrm{q}}=\left[\begin{array}{llll}0 & 0 & -41.8 & -69.6\end{array}\right.$

$\mathrm{a}_{4 \mathrm{q}}=\left[\begin{array}{llllll}0 & 0 & 0 & 0 & 85.5\end{array}\right.$

$-39.2]$

$\mathrm{a}_{6 \mathrm{q}}=\left[\begin{array}{lllll}0 & 0 & 0 & 0 & 0\end{array}\right.$

$\begin{array}{llll}173.6 & 158.0 & 63.3 & -29.6\end{array}$

$\begin{array}{lllllllll}0 & 0 & 222.0 & 112.4 & -189.8 & -291.8 & 370.5 & -372.8 & 296.5]\end{array}$

pling constant, the following energies of the seven Kramers doublets (Table 6) are obtained:

Table 6. Energies of the Kramers doublets of $\mathrm{Yb}\left(\mathrm{NO}_{3}\right)_{5}^{-}$

\begin{tabular}{|l|c|c|}
\hline Spinor state & $\mathrm{E}_{\text {pred }}\left[\mathrm{cm}^{-1}\right]$ & $\mathrm{E}_{\text {obs }}\left[\mathrm{cm}^{-1}\right]$ \\
\hline $\mathrm{E}^{\prime}\left({ }^{2} \mathrm{~F}_{7 / 2}\right)$ & 0 & 0 \\
\hline $\mathrm{E}^{\prime}\left({ }^{2} \mathrm{~F}_{7 / 2}\right)$ & 146 & 12 (outlier?) \\
\hline $\mathrm{E}^{\prime}\left({ }^{2} \mathrm{~F}_{7 / 2}\right)$ & 255 & 60 \\
\hline $\mathrm{E}^{\prime}\left({ }^{2} \mathrm{~F}_{7 / 2}\right)$ & 461 & 270 \\
\hline $\mathrm{E}^{\prime}\left({ }^{2} \mathrm{~F}_{5 / 2}\right)$ & $10^{\prime} 582$ & $10^{\prime} 438$ \\
\hline $\mathrm{E}^{\prime}\left({ }^{2} \mathrm{~F}_{5 / 2}\right)$ & $10^{\prime} 636$ & $10^{\prime} 534$ \\
\hline $\mathrm{E}^{\prime}\left({ }^{2} \mathrm{~F}_{5 / 2}\right)$ & $10^{\prime} 759$ & $10^{\prime} 604$
\end{tabular}

We analyze the Zero Field Splitting using the conventional effective Spin Hamiltonian (Eqn. (33)):

$$
\hat{H}_{Z F S}^{e f f}=D\left[S_{z}^{2}-\frac{1}{3} S(S+1)\right]+E\left[S_{x}^{2}-S_{y}^{2}\right]
$$

The best fit yields: $\mathrm{D}=33.78 \mathrm{~cm}^{-1} ; \mathrm{E}=$ $7.85 \mathrm{~cm}^{-1}$, error ${ }_{\max }=6.1 \mathrm{~cm}^{-1}$, which means that the magnetic anisotropy is quite large.

Moreover, we can easily calculate the Zeeman splitting: $\hat{H}_{Z e}=k_{\text {orb }} \vec{L}+2.0023 \vec{S}$ that is usually analyzed in terms of an effective Spin-Hamiltonian: $\hat{H}_{Z e}^{\text {eff }}=\vec{H} \cdot \mathbf{g} \cdot \vec{S}^{\text {eff }}$

This yields the following g-tensor in the local frame (Table 7). However, in experimental measurements, the diagonal part of the symmetrized square $\mathbf{g}_{\text {sym }}^{2}=\mathbf{g} \cdot \mathbf{g}^{T}$ is observed (Table 7b). Experimentally it is possible to measure the angle $\Delta \phi$ between the indicated $\mathrm{g}$ direction and the $a$ crystal axis in the $a c$ plane. Thus, we give Eigenrotation that diagonalizes $\mathrm{g}^{2}$ in Table $7 \mathrm{c}$.
Table 7. a) $\mathbf{g}$-tensor of $\mathrm{Yb}\left(\mathrm{NO}_{3}\right)_{5}{ }^{-}$in the local frame; b) Symmetrized $\mathbf{g}$-tensor of $\mathrm{Yb}\left(\mathrm{NO}_{3}\right)_{5}^{-}$; c) Eigenvectors that diagonalizes the symmetrized $\mathbf{g}$-tensor in the local frame in a)

a)

$\begin{array}{ccc}\mathrm{X} & \mathrm{z} & \mathrm{y} \\ -2.3684 & -0.0000 & 0.0472 \\ -0.0000 & 2.5308 & 0.0000 \\ -2.7615 & -0.0000 & 4.1083\end{array}$

b)

$$
\mathbf{g}_{\text {sym }}=\left[\begin{array}{ccc}
1.8592 & 0 & 0 \\
0 & 2.5308 & 0 \\
0 & 0 & 5.1632
\end{array}\right]
$$

c)

$$
\mathbf{c}=\left[\begin{array}{ccc}
0.9524 & 0 & 0.3047 \\
0 & 1 & 0 \\
-0.3047 & 0 & 0.9524
\end{array}\right]
$$

From this we obtain angle $\Delta \phi_{\text {pred }}=\cos ^{-1}$ $(0.9524) \cdot 180 / \pi=17.75^{\circ}\left(\Delta \phi_{\text {obs }}=25^{\circ} \pm 5^{\circ}\right)$. These results are displayed in Table 8 .

Finally, in Fig. 7 we give the electronic spectrum and energy level diagram of $\mathrm{Yb}^{3+}$ in $\left[\left(\mathrm{C}_{6} \mathrm{H}_{5}\right)_{4} \mathrm{As}\right]_{2} \mathrm{Yb}\left(\mathrm{NO}_{3}\right)_{5}$.

\section{Example 2: The Prediction of Future Domestic Lighting ${ }^{[12]}$}

We consider here the optical properties of the $\mathrm{Pr}^{3+}$ ion embedded into various solidstate fluoride host lattices, for the prospection and understanding of the so-called quantum cutting process, being important in the further quest of warm-white light source in light emitting diodes (LED). We use LFDFT as described abundantly in the methodological part including the calculations of the Slater-Condon parameters, the ligand field interaction and the spin-orbit coupling constants, important for the nonempirical prediction of future domestic lighting. The model is intended to contribute to the design of modern phosphors and to help to complement the understanding of the $4 \mathrm{f}^{\mathrm{n}} \rightarrow 4 \mathrm{f}^{\mathrm{n}-1} 5 \mathrm{~d}^{1}$ transitions in any lanthanide system.

Next, we calculate the Slater-Condon parameters directly from the radial functions of the $4 \mathrm{f}$ and $5 \mathrm{~d}$ Kohn-Sham orbitals. We report here the reliable prediction and understanding of the $4 \mathrm{f}^{\mathrm{n}} \rightarrow 4 \mathrm{f}^{\mathrm{n}-1} 5 \mathrm{~d}^{1}$ transitions considering luminescent materials like $\mathrm{Pr}^{3+}$-doped fluoride host lattices, in order to provide a prospective description of the optical behavior of modern warm-white light phosphors. Various fluoride host lattices doped with $\mathrm{Pr}^{3+}$ ion are investigated taking into account the local symmetry of the lanthanide center together with its coordination sphere, as

Table 8. Predicted and experimental principal g-values and directions for $\left[\left(\mathrm{C}_{6} \mathrm{H}_{5}\right)_{4} \mathrm{As}\right]_{2} \mathrm{Yb}\left(\mathrm{NO}_{3}\right)_{5}$

$$
\text { Predicted g-value Observed g-value Predicted } \Delta \phi^{\mathrm{a}}
$$

Observed $\Delta \phi^{\mathrm{a}}$

\begin{tabular}{l|l|l|}
$\mathrm{g}_{1}$ & 5.16 & $5.48 \pm 0.03$ \\
$\mathrm{~g}_{2}$ & 2.53 & $2.45 \pm 0.03$ \\
$\mathrm{~g}_{3}$ & 1.86 & $1.76 \pm 0.02$
\end{tabular}
108

$115 \pm 5$

\begin{tabular}{l|l}
$\mathrm{g}_{3}$ & 1.86
\end{tabular}

18

$25 \pm 5$

${ }^{\mathrm{a}} \Delta \phi$ the angle between the indicated $\mathrm{g}$ direction and the $\mathbf{a}$ crystal axis in the ac plane 


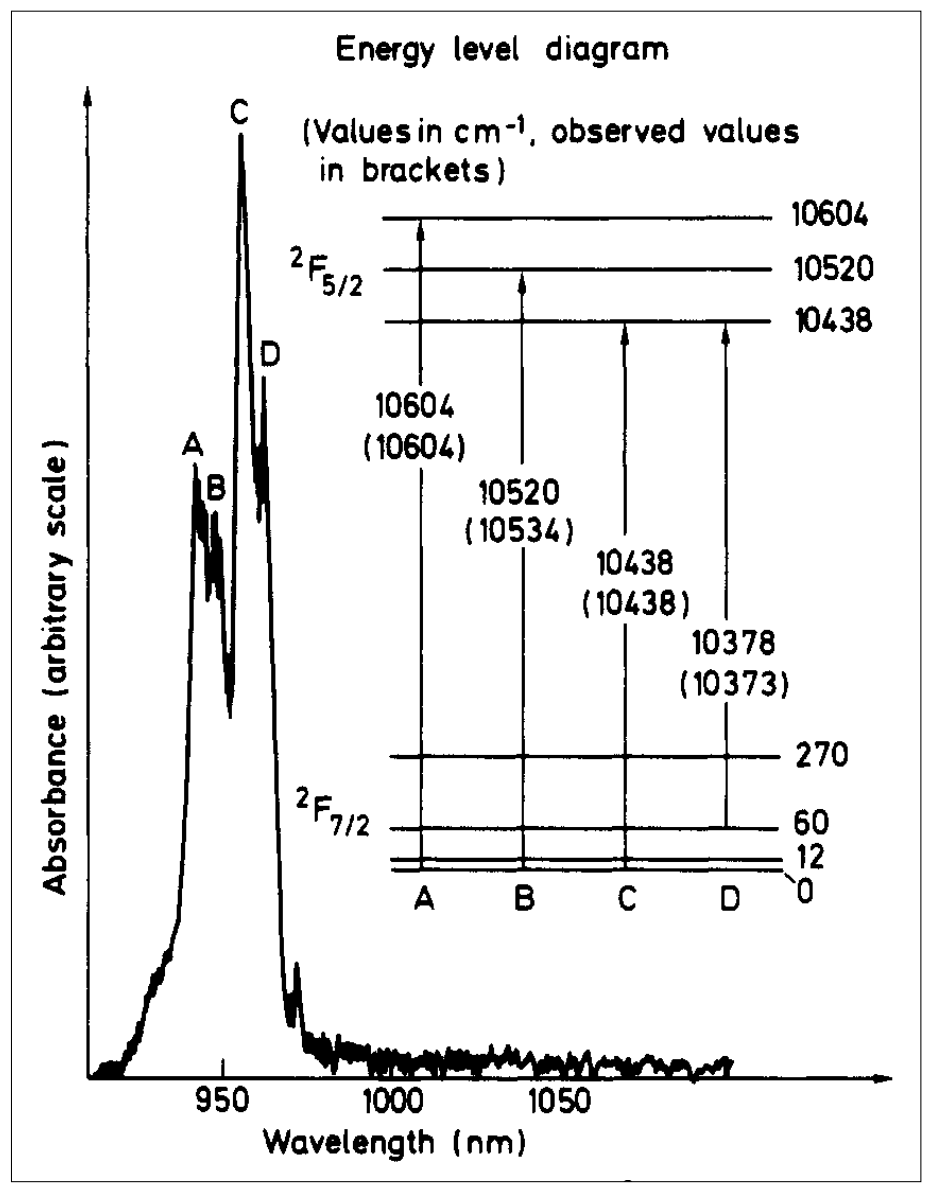

Fig. 7. Single-crystal electronic spectrum and energy level diagram of $\mathrm{Yb}^{3+}$ in $\left[\left(\mathrm{C}_{6} \mathrm{H}_{5}\right)_{4} \mathrm{As}\right]_{2} \mathrm{Yb}\left(\mathrm{NO}_{3}\right)_{5}$. The energies were adjusted using the Angular Overlap Model and are almost identical to our calculated ones.
Fig. 9 shows the predicted LF-splittings in the various environment.

Finally Fig. 10 shows the result of our prediction: (a) and (c) are not quantum cutters, whereas (b) and (d) are. It is interesting to note that in this latter case there will be two photons emitted for on absorbed high-energy photon. The theoretical quantum yield is two. The electrostatic matrixelements elements are obtained considering the nephelauxetic effect already depicted in case of fluoride ligands in Fig. 5.

\section{Conclusion}

The best light source alternative after the banishment on incandescent light bulbs is certainly obtained within simple, inexpensive and energy efficient technology. LED lighting is undoubtedly the future of our domestic lighting. One usually judges the efficiency of the LED with respect to the optical manifestation of the inorganic phosphorus which is used. These inorganic phosphorus compounds, currently based on the doping of lanthanide ion into stable crystal host lattices, gives suitable visible light emission necessary to the generation of warm-white light. As written, nowadays LED lighting is not only understood by the well as the availability of optical spectra. Hence, a special emphasis is addressed to systems, which have been experimentally investigated before, in order to validate the theoretical approach. In this respect, we choose $\mathrm{Pr}^{3+}$ coordinated with eight fluoride ligands as experimentally observed in the following systems: $\mathrm{CaF}_{2}: \mathrm{Pr}^{3+}, \mathrm{KY}_{3} \mathrm{~F}_{10}: \mathrm{Pr}^{3+}$, $\mathrm{BaY}_{2} \mathrm{~F}_{8}: \mathrm{Pr}^{3+}$ and $\mathrm{LaZrF}_{7}: \operatorname{Pr}^{3+}$, where the eight fluoride ligands form arrangement with $\mathrm{O}_{\mathrm{h}}, \mathrm{C}_{4 \mathrm{v}}, \mathrm{C}_{2}$ and $\mathrm{C}_{1}$ symmetry, respectively (Fig. 8).

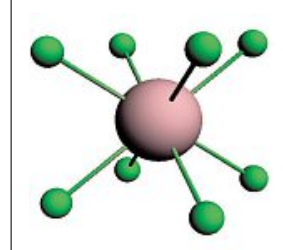

(a)

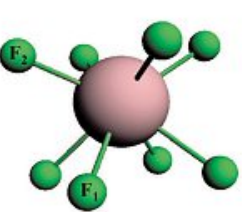

(b)

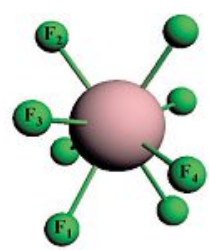

(c)

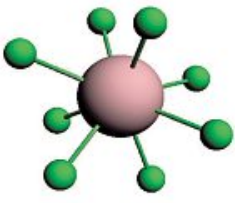

(d)
Fig. 8. Structure and spatial representation of the eight coordinated $\mathrm{Pr}^{3+}$ complexes being investigated in the present work, resulting from $\mathrm{Pr}^{3+}$ doped into: $\mathrm{CaF}_{2}(\mathrm{a}), \mathrm{KY}_{3} \mathrm{~F}_{10}(\mathrm{~b}), \mathrm{BaY}_{2} \mathrm{~F}_{8}$ (c) and $\mathrm{LaZrF}_{7}$ (d) forming a local symmetry of $\mathrm{O}_{\mathrm{h}}, \mathrm{C}_{4 \mathrm{v}}, \mathrm{C}_{2}$ and $\mathrm{C}_{1}$ point groups, respectively.

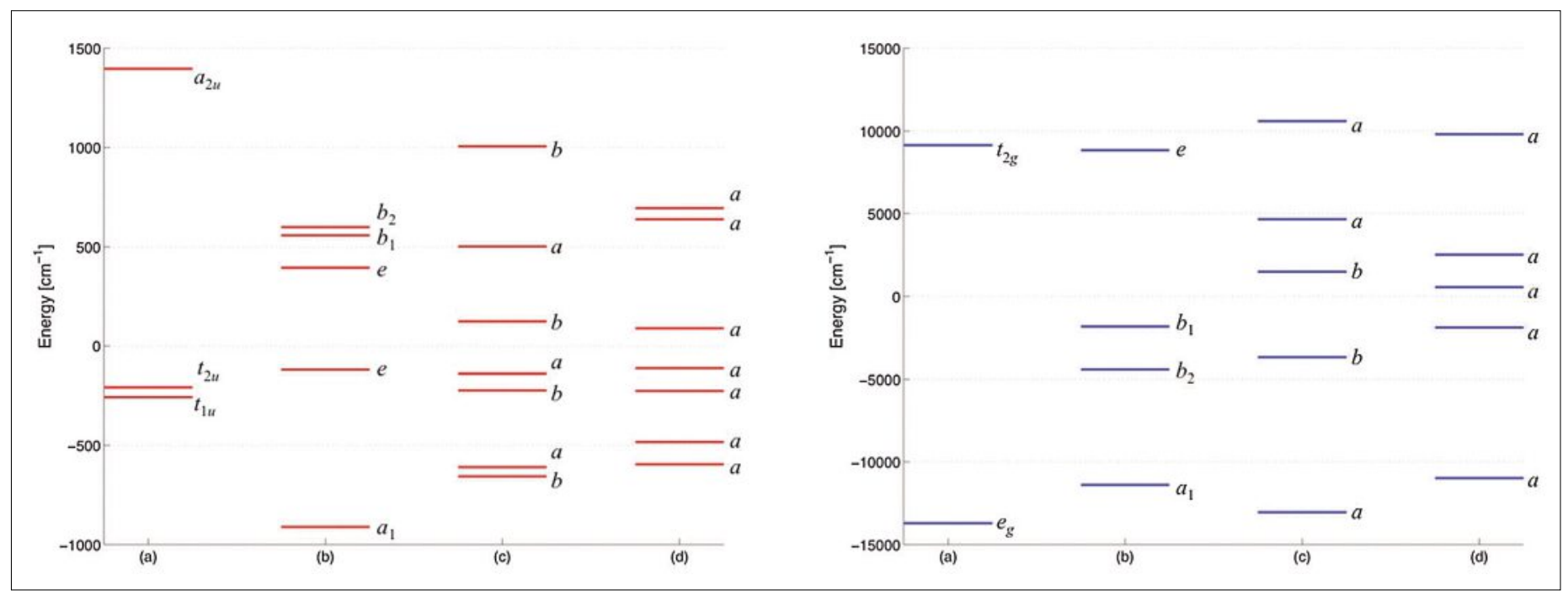

Fig. 9. Calculated energy splitting in $\mathrm{cm}^{-1}$ of the $4 \mathrm{f}$ (left hand side, in red) and the $5 \mathrm{~d}$ (right hand side, in blue) orbitals of $\mathrm{Pr}^{3+}$ due to ligand field interaction in the system: $\mathrm{CaF}_{2}: \mathrm{Pr}^{3+}(\mathrm{a}), \mathrm{KY}_{3} \mathrm{~F}_{10}: \mathrm{Pr}^{3+}(\mathrm{b}), \mathrm{BaY}_{2} \mathrm{~F}_{8}: \mathrm{Pr}^{3+}$ (c) and $\mathrm{LaZrF}_{7}: \mathrm{Pr}^{3+}$ (d). The irreducible representations of the point group under which the local structures of the $\mathrm{Pr}^{3+}$ impurity belong, are also indicated. 


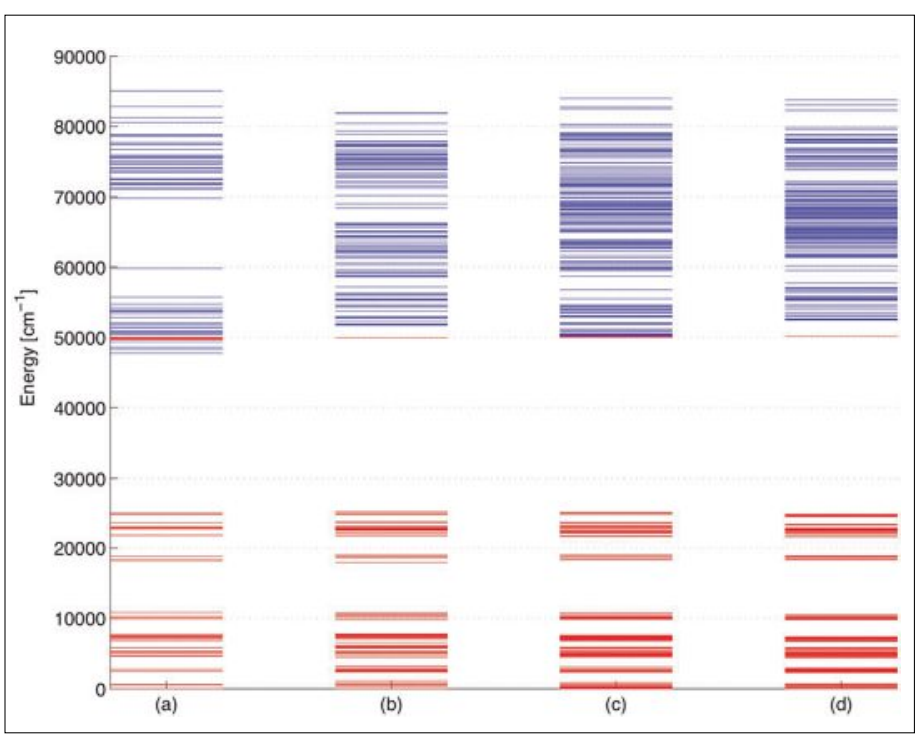

Fig. 10 Calculated multiplet energy levels in $\mathrm{cm}^{-1}$ obtained for $\mathrm{Pr}^{3+}$ doped into $\mathrm{CaF}_{2}(\mathrm{a}), \mathrm{KY}_{3} \mathrm{~F}_{10}$ (b), $\mathrm{BaY}_{2} \mathrm{~F}_{8}$ (c) and $\mathrm{LaZrF}_{7}$ (d). The energies of the terms originating from the ground electron configuration of $\operatorname{Pr}^{3+}\left(4 f^{2}\right)$ are in red, those for the excited configuration $\left(4 f^{1} 5 d^{1}\right)$ are in blue.

analysis of the $\mathrm{f}$ - $\mathrm{d}$ dipole allowed transitions in the lanthanide system, but also limited to the bluish cold-white light emission of the $\mathrm{Ce}^{3+}$-based LED bulbs.

Lanthanides like $\mathrm{Pr}^{3+}$ and $\mathrm{Eu}^{2+}$ are already undeniably conceived as activators of warm-white light source, ergo solution for the improvement of LED technology. However, the development and engineering of such a technology are still complex, relying on the perfect understanding of the $4 \mathrm{f}^{\mathrm{n}} \rightarrow 4 \mathrm{f}^{\mathrm{n}-1} 5 \mathrm{~d}^{1}$ transitions. In this paper, we present a fully non-empirical determination of the $4 \mathrm{f}^{\mathrm{n}} \rightarrow 4 \mathrm{f}^{\mathrm{n}-1} 5 \mathrm{~d}^{1}$ transitions in lanthanide doped into crystal host lattices; in order to understand the optical manifestation of new generation of LED phosphors. The method is particularly applied to understand the phenomenology of quantum cutting process in the optical ride lattices. We address the problem in a transparent and clear approach, taking advantages of the facilities given by ligand field theory in conjunction with modern quantum chemistry tools. The proposed LFDFT shows here to be a useful tool, providing reliable and accurate predictions in the perspective to design the optical properties of materials by Density Functional Theory.

Also the analysis of the optical and ESR spectra of low-symmetrical $\left.\left[\mathrm{C}_{6} \mathrm{H}_{5}\right)_{4} \mathrm{As}\right]_{2} \mathrm{Yb}\left(\mathrm{NO}_{3}\right)_{5}$ we have clearly demonstrated that it is not needed to assume idealized high symmetry. The LFDFT model has shown to be a valuable tool for the interpretation of the spectral and magproperties of $\mathrm{Pr}^{3+}$-doped into various fluo- netic properties of low-symmetry rareearth complexes. The predicted values for the EPR parameters and for the Zero Field Splitting of the Kramers doublets gives a valuable insight into chemical bonding between rare-earth ions and ligand ions.

Received: July 2, 2014

[1] C. Daul, J. Phys.: Conf. Ser. 2013, 428, 012023.

[2] C. E. Schäffer, C. K. Jorgensen, Mol. Phys. 1965, 9,401

[3] a) M. Atanasov, C. A. Daul, C. Rauzy, Chem. Phys. Lett. 2003, 367, 737; b) M. Atanasov, C. A. Daul, C. Rauzy, Structure \& Bonding 2004, 106, 97.

[4] A. Borel, C. A. Daul, L. Helm, Chem. Phys. Lett. 2004, 383, 584.

[5] M. Atanasov, E. J. Baerends, P. Baettig, R. Bruyndonckx, C. Daul, C. Rauzy, M. Zbiri, Chem. Phys. Letters 2004, 399, 433.

[6] a) M. Atanasov, C. A. Daul, Chem. Phys. Lett. 2003, 379, 209; b) M. Atanasov, C. A. Daul, Chem. Phys. Lett. 2003, 381, 584

[7] F. Senn, C. A. Daul, J. Molec. Struct.: THEOCHEM 2010, 954, 105

[8] H. Ramanantoanina, W. Urland, F. Cimpoesu, C. Daul, Phys. Chem. Chem. Phys. 2013, 15, 13902.

[9] T. A. Wesolowski, A. Warshel, J. Phys. Chem. 1994, 98, 5183.

[10] R. Latter, Phys. Rev. 1955, 99, 510

[11] W. Urland, R. Kremer, Inorg. Chem. 1984, 23, 1550.

[12] H. Ramanantoanina, W. Urland, F. Cimpoesu, C. Daul, Phys. Chem. Chem. Phys. 2014, 16, 14625 . 